\title{
The gene expression of NGAL and TLR9 in glomerulus and tubulo-interstitium of patients with lupus nephritis
}

\author{
Jianxin Lu ${ }^{1,3}$, Bonnie Ching-Ha Kwan ${ }^{1}$, Fernand Mac-Moune Lai ${ }^{2}$, Paul Cheung-Lung Choi ${ }^{2}$, \\ Lai-Shan Tam ${ }^{1}$, Edmund Kwok-Ming Li ${ }^{1}$, Kai-Ming Chow ${ }^{1}$, Gang Wang ${ }^{1,4}$, Philip Kam-Tao Li ${ }^{1}$, \\ Cheuk-Chun Szeto ${ }^{1}$
}

\footnotetext{
${ }^{1}$ Department of Medicine \& Therapeutics and ${ }^{2}$ Department of Anatomical \& Cellular Pathology, Prince of Wales Hospital, The Chinese University of Hong Kong, Shatin, Hong Kong, China;

${ }^{3}$ Department of Nephrology, Shanghai Ninth People's Hospital, Shanghai Jiao Tong University School of Medicine, Shanghai, China;

${ }^{4}$ Division of Nephrology, Renmin Hospital of Wuhan University, Wuhan, China.

Email: ccszeto@cuhk.edu.hk
}

Received 10 December 2010; revised 15 February 2011; accepted 17 February 2010

\begin{abstract}
Background: The role of neutrophil gelatinase associated lipocalin (NGAL) and Toll-like receptor 9 (TLR9) in the pathogenesis of lupus nephritis remain elusive. Methods: We quantified the glomerular and tubulointerstitial mRNA expression of NGAL and TLR9 in 42 patients with lupus nephritis (LN group) and 10 controls. Results: As compared to controls, LN group had higher glomerular expression of TLR9, and higher tubulointerstitial expression of NGAL and TLR9. Tubulointerstitial NGAL expression significantly correlated with proteinuria $(r=0.492 ; p=$ $0.003)$, renal function $(r=-0.386 ; p=0.022)$ and histological chronicity index $(r=0.540 ; p=0.004)$. Proteinuria had significant correlation with glomerular $(r=0.554 ; p=0.001)$ and tubulointerstitial $(r=0.379$; $p=0.043$ ) TLR9 expression. Furthermore, there was a significant difference in tubulointerstitial expression of NGAL between treatment response groups. Conclusion: There is an increase in intra-renal mRNA expression of NGAL and TLR9 in LN. Although tubulointerstitial expression of NGAL does not correlate with systemic disease activity, it correlates with proteinuria, renal function, and therapeutic response. The role of NGAL in the pathogensis in $L N$, as well as its application as biomarker for lupus nephritis, requires further study.
\end{abstract}

Keywords: Lupus Nephritis; Immunology; Cytokine

\section{INTRODUCTION}

Systemic lupus erythematosus (SLE) is a multisystem autoimmune disease characterized by aberrant cytokines milieu. Although almost all organs in the body could be involved, lupus nephritis (LN) remains the leading cause of morbidity and mortality in SLE. The precise pathogenic mechanism of SLE and LN, however, is not completely understood. Cytokine aberration is a cardinal phenomenon of LN. It is important to realize that cytokines may not only be involved in the generation of aberrant immune regulation [1], but also participates in the local inflammatory processes that ultimately lead to tissue destruction. Unfortunately, most studies focused on the cytokine profile of peripheral blood in SLE patients, and the results are often inconsistent [2,3]. Since specific organ or tissue involvement in SLE probably involve local cytokine aberrations that do not appear in the systemic circulation, study of the immunopathogenesis should focus at the specific sites of disease involvement.

Neutrophil gelatinase associated lipocalin (NGAL) is a member of the lipocalin superfamily, which is first found in the specific granules of human neutrophil [4]. NGAL is a promising biomarker for both acute kidney injury (AKI) and chronic kidney disease (CKD) [5]. Urinary NGAL levels correspond to disease activity in lupus nephritis more closely than standard markers [6]. Toll-like receptor 9 (TLR9) is a pattern-associated receptor functioning in innate immunity that recognizes unmethylated $\mathrm{CpG}$ sequences in DNA molecules; it may be involved in the recognition of self DNA-antigens and the production of pathogenic auto-antibodies. Previous study showed that a higher expression of TLR9 on peripheral blood B cells from patients with active SLE correlated with SLEDAI [7]. Notably, TLR9 causes the activation of NF- $\kappa B$ [8], which is recruited to the NGAL promoter and is essential for the transcriptional activa- 
tion of NGAL promoters [9]. We hypothesize that intra-renal TLR9 and NGAL are related to the development of LN.

\section{PATIENTS AND METHODS}

\subsection{Patient Selection}

We studied 42 consecutive SLE patients with active renal disease and required kidney biopsy. All patients fulfilled the American College of Rheumatology diagnostic criteria of SLE [10]. The uninvolved pole of 10 kidneys that were removed for renal cell carcinoma and had no morphological evidence of renal disease were used as control. The study was approved by the Clinical Research Ethical Committee of the Chinese University of Hong Kong; all patients provided informed consent.

\subsection{Clinical and Histological Assessment}

The disease activity of SLE was assessed clinically by the Systemic Lupus Erythematosus Disease Activity Index (SLEDAI) [11] on the day of kidney biopsy by an independent physician. Baseline serum creatinine, urine protein, complement levels ( $\mathrm{C} 3$ and $\mathrm{C} 4)$ and anti-double strand (ds) DNA antibody titre were also measured. Glomerular filtration rate (GFR) was estimated by a standard equation [12].

Kidney biopsy specimen was evaluated according to the International Society of Nephrology (ISN) classifycation of lupus nephritis [13]. The activity index (AI) and chronicity index (CI) of each biopsy specimen were scored by standard methods [14]. Briefly, AI is the sum of semiquantitative manual scores (0-3 each) of the following parameters: Endocapillary hypercellularity; leucocyte infiltration, subendothelial hyaline deposits; interstitial inflammation, necrosis and cellular crescents. Scores of the last two parameters are counted double, making a total AI of 0 to 24 .

\subsection{Laser Microdissection}

The method of laser microdissection has been described in our previous studies $[14,15]$. Briefly, cryosections of $10 \mu \mathrm{m}$ thickness were prepared on a cryostat (Leica Microsystems, Wetzlar, Germany) using disposable microtome blades (Leica Microsystem) in RNase-free conditions, and were mounted on MembraneSlide 0.17 PEN slides (Carl Zeiss PALM Microlaser Technologies, Bernried, Germany). Immediately after taking the slides out of the cryostat, the sections were fixed in $70 \%$ ethanol and dehydrated in $100 \%$ ethanol. Sections were airdried at room temperature.

Laser microdissection of the snap-frozen kidney biopsy specimens was performed using the PALM Microlaser System (PALM Microlaser Technologies), which is equipped with a pulsed high-quality laser beam, computer-controlled microscope stage and micromanipulator. Under direct visual control, areas of interest in the histological specimens were selected through the PALM RoboSoftware (PALM Microlaser Technologies) by moving the computer mouse, and microdissected by cutting the contour of the selected areas with the adjusted laser beam. The isolated tissue was then lasercatapulted into a microcentrifuge cap filled with guanidine thiocyanate containing lysis buffer for the subsequent RNA isolation. Approximately 20-30 glomerulus and 20 randomly selected tubulointerstitial area were isolated from each specimen. The tissue lysate of glomerulus and tubulointerstitium were kept at $-80^{\circ} \mathrm{C}$ until RNA extraction was performed with the RNAqueous ${ }^{\circledR}$-Micro Kit (Applied Biosystems, USA), following manufacturer's instruction.

\subsection{Quantification of Intrarenal Gene Expression}

The method of total RNA extraction, reverse transcription and real-time quantitative polymerase chain reaction (RT-QPCR) was described in our previous studies $[14,16]$. In the present work, we quantified the mRNA expression of NGAL and TLR9 in glomerulus and tubulointerstitium. Taqman primers and probes of each target were purchased from Applied Biosystems (Foster City, California, USA). The RT-QPCR was performed by ABI Prism 7900HT Sequence Detector System (Applied Biosystems) following the manufacturer's instruction. The mRNA expression for each signal was calculated by using the $\Delta \mathrm{Ct}$ procedure according to manufacturer's instruction, with glyceraldehyde-3-phosphate dehydrogenase (GAPDH) used as the housekeeping gene for normalisation among samples. All primers and probes were tested with purified DNA as the template in RT-QPCR to ensure that they do not amplify genomic DNA. All results were analysed by Sequence Detection Software version 1.7 (Applied Biosystems).

Gene expression of each target was calculated by using the difference-in-threshold-cycle $(\Delta \mathrm{Ct})$ procedure, according to manufacturer's instruction. For GAPDH and each target, the relative efficiency of amplification over various starting template concentrations was determined. Approximately equal efficiencies for other targets with GAPDH amplifications were verified by an absolute value of $<0.1$ for the slope of log input complementary DNA amount versus $\Delta \mathrm{Ct}$, which was obtained by subtracting the threshold cycle $(\mathrm{Ct})$ value of GAPDH from that of the target. Therefore, it was possible to detect GAPDH in the same tube with other targets. The relative quantification of using multiplex reaction with a comparative method was determined by the formula $2^{-(\Delta \Delta \mathrm{Ct})}$, where the $\Delta \Delta \mathrm{Ct}$ was calculated by the subtrac- 
tion of $\Delta \mathrm{Ct}$ of the calibrator from $\Delta \mathrm{Ct}$ of the sample.

\subsection{Clinical Follow-Up}

After the renal biopsy, all patients were followed for at least 6 months. The clinical management was decided by individual nephrologist and not affected by the study. In general, patients were treated with corticosteroid, together with cyclophosphamide or mycophenolate according to published protocols. Therapeutic response to induction immunosuppressant was assessed at 12 weeks after treatment and classified into complete remission, partial remission, and no response as defined previously [17]. Briefly, complete remission was defined as a value for urinary protein excretion that was less than $0.3 \mathrm{~g} / 24$ hours, with normal urinary sediment, a normal serum albumin concentration, and values for both serum creatinine and estimated GFR that were $15 \%$ or less above the base-line values. Partial remission was defined as a value for urinary protein excretion that was between 0.3 and $2.9 \mathrm{~g} / 24$ hours, with a serum albumin concentration of at least $30 \mathrm{~g} / \mathrm{dL}$ and stable renal function. Treatment failure was defined as a value for urinary protein excretion that remained at or above $3 \mathrm{~g} / 24$ hours or a value of 0.3 to $2.9 \mathrm{~g} / 24$ hours but with a serum albumin below 30 $\mathrm{g} / \mathrm{dL}$, an increase in the serum creatinine concentration $\geq$ $50 \mu \mathrm{mol} / 1$, or a value for estimated GFR that was more than $15 \%$ above the base-line value, or the discontinuation of treatment due to side effects.

\subsection{Statistical Analysis}

Statistical analysis will be performed by SPSS for Windows software version 15.0 (SPSS Inc., Chicago, IL). All the results from this series of experiments are quantitative. The results are presented as mean \pm SD unless otherwise specified. Since the data on gene expression are highly skewed, they are compared between groups by Kruskall Wallis test or Mann-Whitney U test as appropriate. Correlations between continuous variables are calculated by Spearman's rank correlation coefficient. A $\mathrm{p}$ value of less than 0.05 is considered as statistically significantly. All probabilities are two tailed.

\section{RESULT}

We studied 42 SLE patients. Their baseline demographic and clinical data are summarized in Table 1. The histological diagnoses were proliferative nephritis (class III or IV, 9 cases), pure membranous nephritis (class V, 9 cases), class II nephritis (3 cases), and mixed proliferative and membranous nephritis (21 cases). The mean histological Activity and Chronicity Indices were $7.1 \pm$ 4.3 and $2.7 \pm 2.2$, respectively.
Table 1. Baseline demographic and clinical data.

\begin{tabular}{ccc}
\hline & Patient group & Control group \\
\hline No. of patient & 40 & 10 \\
Sex (M:F) & $2: 40$ & $4: 6$ \\
Age (years) & $40.8 \pm 11.4$ & $36.4 \pm 13.7$ \\
SLEDAI score & $7.6 \pm 4.5$ & 0 \\
Serum creatinine ( $\mu \mathrm{mol} / \mathrm{l})$ & $125.4 \pm 115.5$ & $82.7 \pm 14.0$ \\
Proteinuria (g/day) & $2.33 \pm 1.84$ & $0.01 \pm 0.03$ \\
Glomerular filtration rate & $71.2 \pm 37.2$ & $106.6 \pm 22.0$ \\
(mL/min/1.73m $\left.{ }^{2}\right)$ & & \\
Renal histology & $13.8 \pm 17.9$ & $1.4 \pm 2.344$ \\
Interstitial fibrosis (\%) & $10.8 \pm 15.5$ & $2.6 \pm 5.3$ \\
Glomerosclorosis (\%) &
\end{tabular}

SLEDAI, Systemic Lupus Erythematosus Disease Activity Index.

\subsection{Gene Expression Level between Groups}

The glomerular and tubulointerstitial mRNA expression levels of NGAL and TLR9 are summarized in Figure 1. As compared to controls, LN patients had higher glomerular expression of TLR9 $(p<0.001)$ but not NGAL. In contrast, LN patients had higher tubulointerstitial expression of TLR9 $(\mathrm{p}=0.011)$ and NGAL $(\mathrm{p}<0.001)$ as compared to controls. There was a modest internal correlation between tubulointerstitial mRNA expression levels of NGAL and TLR9 $(r=0.386 ; p=0.047)$. The glomerular and tubulointerstitial expression of NGAL are also significantly correlated $(r=0.410 ; p=0.03)$, while those of TLR9 had no significant correlation $(\mathrm{r}=$ $0199, \mathrm{p}=0.388$ ). There were no significant differences in glomerular or tubulointerstitial expression of TLR9 or NGAL between histological groups (details not shown).

\subsection{Relation with Clinical and Histological Parameters}

We further explore the correlation between gene expression and baseline clinical and histological parameters within patients with lupus nephritis. We found that tubulointerstitial, but not glomerular, NGAL significantly correlated with proteinuria $(\mathrm{r}=0.492 ; \mathrm{p}$ $=0.003)$, estimated GFR $(\mathrm{r}=-0.386 ; \mathrm{p}=0.022)$ and histological CI $(\mathrm{r}=0.54 ; \mathrm{p}=0.004)$ (Figure 2). In contrast, both glomerular $(\mathrm{r}=0.554 ; \mathrm{p}=0.001)$ and tubulointerstitial ( $r=0.379 ; \mathrm{p}=0.043$ ) TLR9 expression significantly correlated with proteinuria, but not estimated GFR or histological CI (Figure 3). The glomerular or tubulointerstitial expressions of TLR9 or NGAL did not correlate with markers of systemic disease activity, including SLEDAI score, serum complement, or anti-ds DNA titre (details not shown). 
(a)

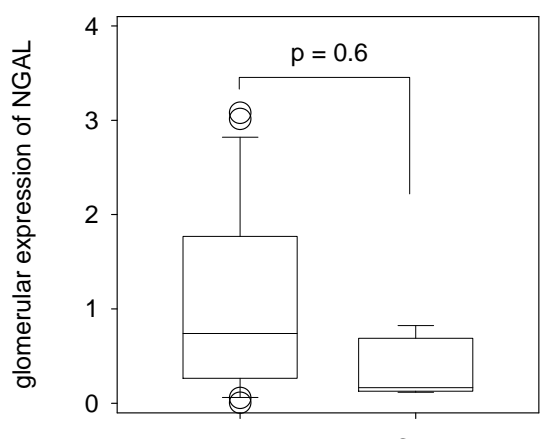

(c)

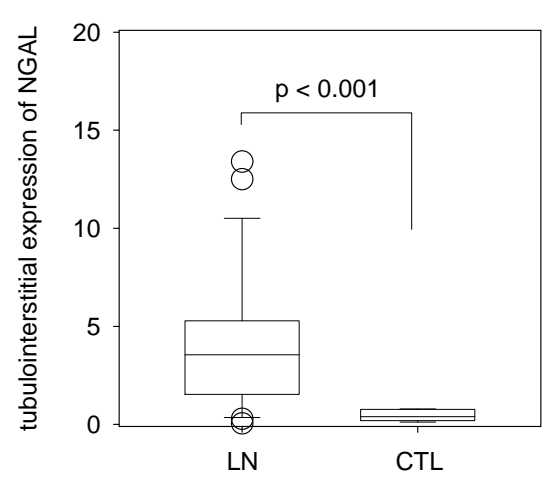

(b)

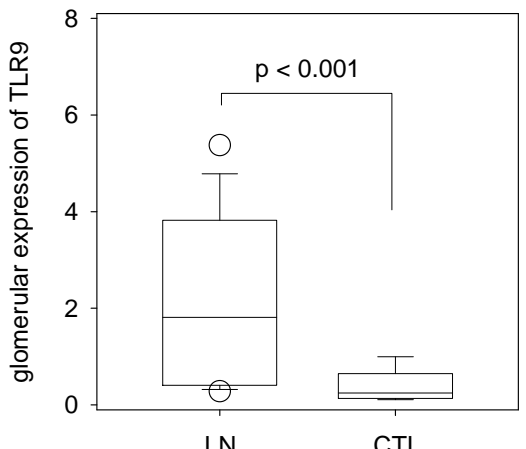

(d)

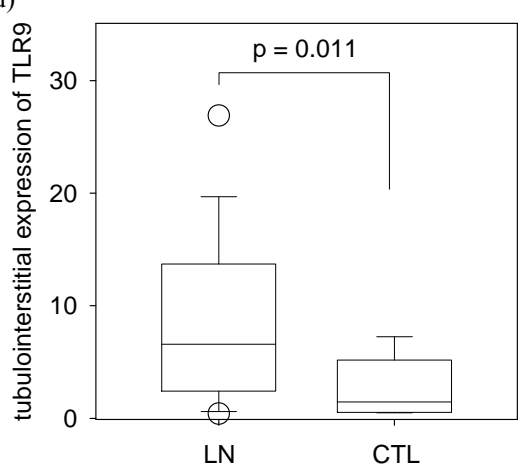

Figure 1. Comparison of glomerular expression of (a) NGAL and (b) TLR9, and tubulointerstitial expression of (c) NGAL and (d) TLR9, between groups. Whisker-box plot, with the boxes indicate median, 25 and 75 percentile; whisker caps indicate 5 and 95 percentile; circles indicate outliers. Data are compared by Mann-Whitney U test. (LN, lupus nephritis group; CTL, control group)

(a)
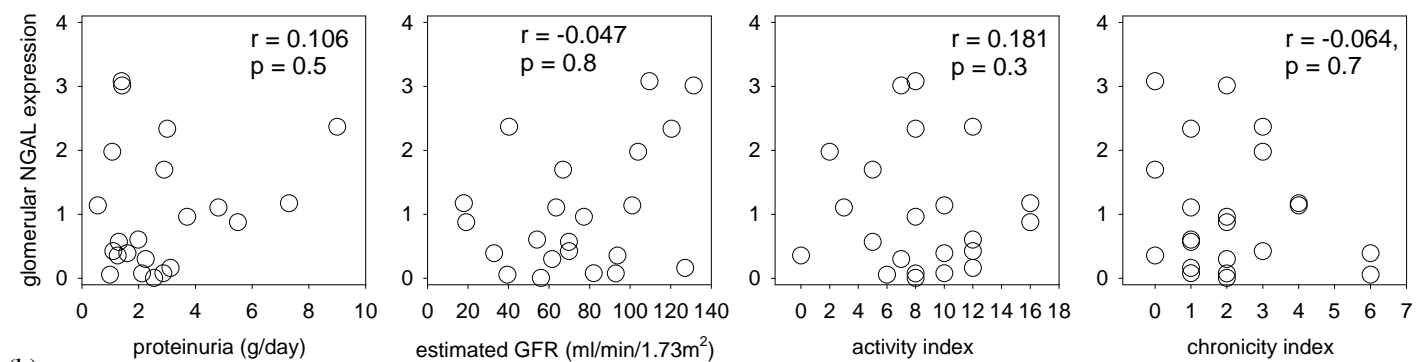

(b)
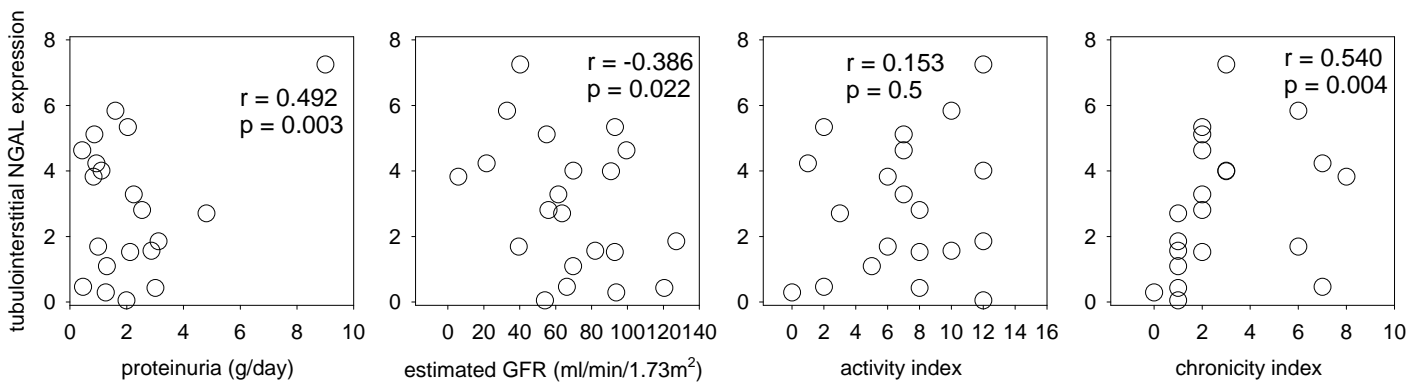

Figure 2. Correlation between (a) glomerular and (b) tubulointerstitial expression of NGAL and the degree of proteinuria, estimated glomerular filtration rate (GFR), histological activity index and chronicity index. Data are compared by Spearman's correlation coefficient. (Some of the circles overlap; each circle on the figure may represent more than one patient.) 
(a)

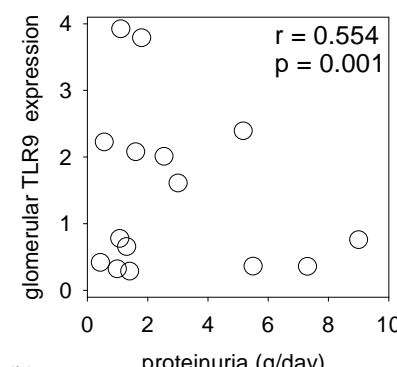

(b)

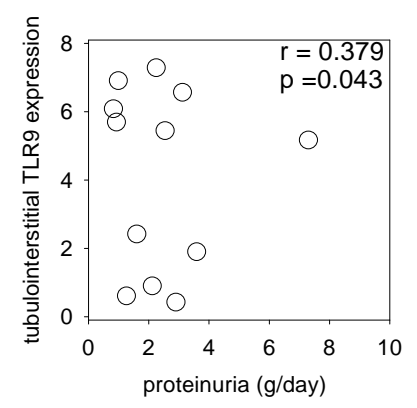

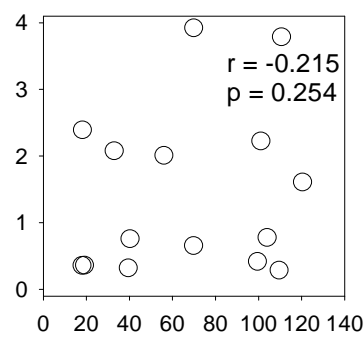

estimated GFR $\left(\mathrm{ml} / \mathrm{min} / 1.73 \mathrm{~m}^{2}\right)$

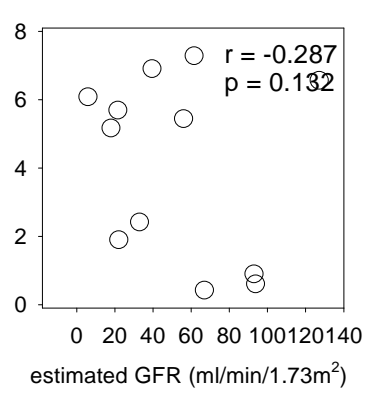

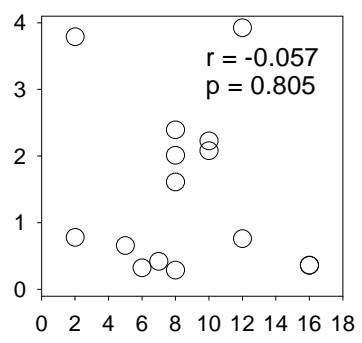

activity index

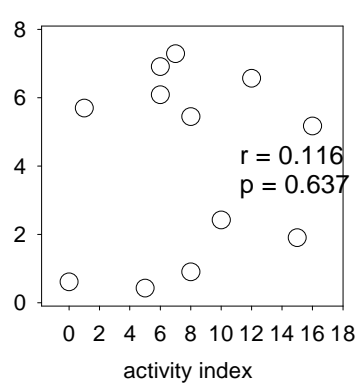

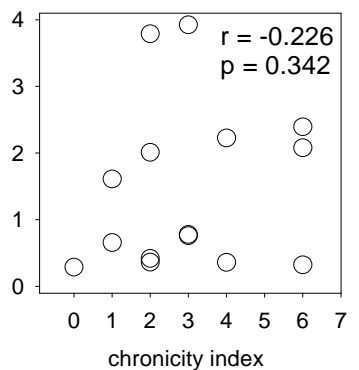

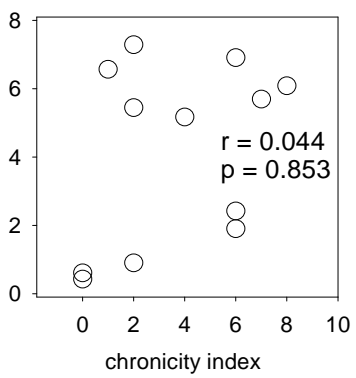

Figure 3. Correlation between (a) glomerular and (b) tubulointerstitial expression of TLR9 and the degree of proteinuria, estimated glomerular filtration rate (GFR), histological activity index and chronicity index. Data are compared by Spearman's correlation coefficient. (Some of the circles overlap; each circle on the figure may represent more than one patient.)

\subsection{Relation with Therapeutic Responses}

After 6 months follow up, 14 cases had complete remission (CR), 21 cases had partial remission (PR) cases and 7 cases had no response (NR). The expressions of the two target genes are compared between treatment response groups (Figure 4). There was a significant difference in tubulointerstitial expression of NGAL between treatment response groups. Post hoc analysis showed that, as compared to CR group, NR group had lower tubulointerstitial expression of NGAL $(p=0.039)$. There was no significant difference in the glomerular expression of NGAL, or glomerular or tubulointerstitial expression of TLR9, because treatment response groups.

\section{DISCUSSION}

In the present study, we found that NGAL expression only increased in the tubulointerstitium of LN. Our result is similar to that of Brunner et al., who found significantly greater urinary NGAL levels in individuals with active renal disease as compared to others [18]. Our findings also suggest that NGAL expression was abundant in tubulointerstitium but not glomerulus, which is consistent with the general belief. Furthermore, we found that tubulointerstitial NGAL expression significantly correlated with proteinuria, GFR and CI. The result agrees with the report of Bolignano et al., who also showed that urinary NGAL concentrations correlated with renal function and proteinuria [19]. In addition, we found that the tubulointerstitial expression of NGAL is related to treatment response. Our results concur with the report of Hinze et al. [20], which also showed that urinary and plasma NGAL are predictors of the course of LN in childhood-onset SLE patients. Taken together, the data suggest that the alteration of tubulointerstitial NGAL in LN is functionally relevant.

The site of renal TLR9 expression has been a matter of debate. In a recent study, Machida et al. [21] reported injured glomerular podocytes in active LN expressed TLR9, which disappeared during remission and was not expressed in a normal control kidney. Furthermore, TLR9 expression correlated with the degree of proteinuria and anti-dsDNA antibody titre. On the other hand, Benigni et al. [22] showed a robust TLR9 expression in the proximal tubular epithelial cells in an animal lupus model and patients with LN, and TLR9 level correlated with proteinuria and tubular damage. Our result showed that TLR9 expression increased in both glomerulus and tubulointerstitium of LN, which is consistent with the report of Papadimitraki et al. [23].

The role of TLR9 in LN is relatively well studied. The up-regulation of TLR9 in the renal parenchyma of LN may be a response to inflammatory cytokines or the nucleosomes present within the lesion [23]. In return, TLR9 induces macrophages and B-cells to produce cytokines and chemokines, thus aggravates inflammation and tissue damage [24-26]. Studies showed that inter 
(a)

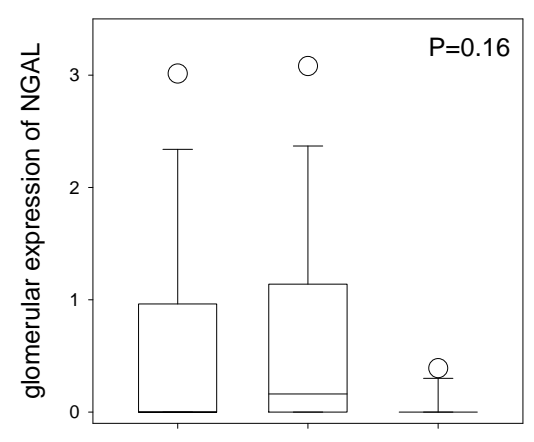

(c)

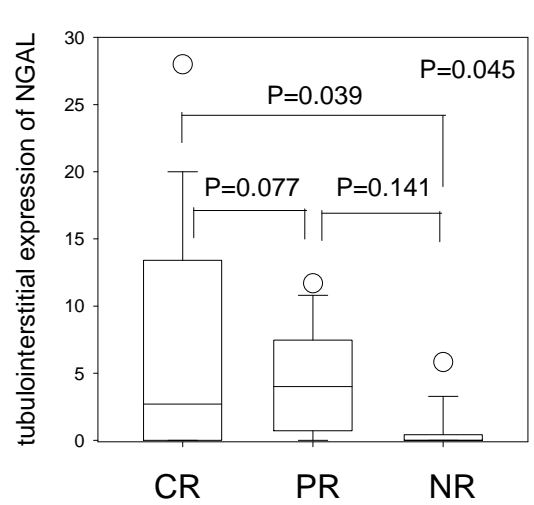

(b)

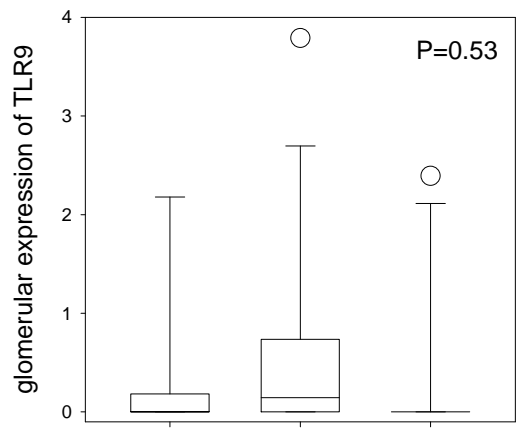

(d)

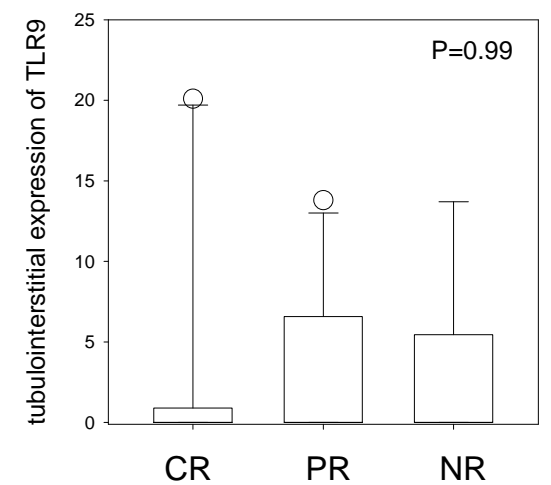

Figure 4. Comparison of glomerular expression of (a) NGAL and (b) TLR9, and tubu lointerstitial expression of (c) NGAL and (d) TLR9, between treatment response groups. Data are compared by Kruskal Wallis test. The box indicates median, 25 and 75 percentile; whisker caps indicate 5 and 95 percentile; outliers are represented as closed circles. (Key for response groups: $\mathrm{CR}$, complete remission; $\mathrm{PR}$, partial remission; NR, no response.)

nalization of SLE immune complexes into subcellular lysosomes containing TLR9 induced a signaling cascade that led to the activation of dendritic cells and production of cytokines and chemokines [27], suggesting that TLR9 may have a role in the onset and progression of LN. In the present study, we found a modest, although statistically significant, correlation between tubulointerstitial expression of NGAL and TLR9. Our result indicates that tubulointerstitial NGAL expression is governed by factors other than TLR9.

Several limitations of the current study need to be addressed. First, the sample size of our study was small, so that subgroup comparison of gene expression between different histological classes of lupus nephritis may not be reliable. Because of the limitated amount of pathological specimen available, we did not quantify intrarenal TLR9 or NGAL at protein level by immunohistochemistry or western blotting. In addition, we could not examine renal tissue of lupus patients without kidney problem as the control group. Furthermore, we do not examine the longitudinal change of intra-renal gene expression or the response to immunosuppressive therapy by serial renal biopsy. Further experiments are necessary to solve these limitations and confirm our findings.

In summary, we found an increase in intra-renal mRNA expression of NGAL and TLR9 in LN. Although tubulointerstitial expression of NGAL does not correlate with systemic disease activity, it correlates with proteinuria, renal function, and therapeutic response. The role of NGAL in the pathogensis in LN, as well as its application as biomarker for lupus nephritis, require further study.

\section{ACKNOWLEDGEMENT}

This study was supported in part by the CUHK research account 6901031. All authors declare no conflict of interest. The results presented in this paper have not been published previously in whole or part, except in abstract format.

\section{REFERENCES}

[1] Lauwerys, B. and Houssiau, F. (1998) Cytokines: Clues to the pathogenesis of SLE. Lupus, 7, 211-213. doi:10.1191/096120398678920136

[2] Karonitsch, T., Feierl, E., Steiner, C., et al. (2009) Activation of the interferon-gamma signaling pathway in systemic lupus erythematosus peripheral blood mono- 
nuclear cells. Arthritis Rheum, 60, 1463-1471. doi:10.1002/art.24449

[3] Lee, H., Mima, T., Sugino, H., et al. (2009) Interactions among type I and type II interferon, tumor necrosis factor, and beta-estradiol in the regulation of immune responserelated gene expressions in systemic lupus erythematosus. Arthritis Res Ther, 11, 1.

[4] Kjeldsen, L., Bainton, D., Sengeløv, H., et al. (1994) Identification of neutrophil gelatinase-associated lipocalin as a novel matrix protein of specific granules in human neutrophils. Blood, 83, 799-807. doi:10.1080/00365510802150158

[5] Devarajan, P. (2008) Neutrophil gelatinase-associated lipocalin (NGAL): A new marker of kidney disease. Scandinavian journal of clinical and laboratory investigation, 241, 89-94.

[6] Pitashny, M., Schwartz, N., Qing, X., et al. (2007) Urinary lipocalin-2 is associated with renal disease activity in human lupus nephritis. Arthritis Rheum, 56, 1894-1903.

[7] Nakano, S., Morimoto, S., Suzuki, J., et al. (2008) Role of pathogenic auto-antibody production by Toll-like receptor 9 of B cells in active systemic lupus erythematosus. Rheumatology, 47, 145-149.

[8] Hemmi, H., Takeuchi, O., Kawai, T., et al. (2000) A Toll-like receptor recognizes bacterial DNA. Nature, 408, 740-745. doi: $10.1038 / 35047123$

[9] Matsuo, S., Yamazaki, S., Takeshige, K., et al. (2007) Crucial roles of binding sites for NF-kappaB and C/EBPs in IkappaB-zeta-mediated transcriptional activation. Biochemical Journal, 405, 605-615. doi:10.1042/BJ20061797

[10] Hochberg, M. (1997) Updating the American college of rheumatology revised criteria for the classification of systemic lupus erythematosus. Arthritis Rheum, 40, 1725. doi:10.1002/art.1780400928

[11] Bombardier, C., Gladman, D., Urowitz, M., et al. (1992) Derivation of the SLEDAI. A disease activity index for lupus patients. The Committee on Prognosis Studies in SLE. Arthritis Rheum, 35, 630-640.

[12] Levey, A., Bosch, J., Lewis, J., et al. (1999) A more accurate method to estimate glomerular filtration rate from serum creatinine: A new prediction equation. Modification of diet in renal disease study group. Annals of Internal Medicine, 130, 461-470.

[13] Weening, J., D'Agati, V., Schwartz, M., et al. (2004) The classification of glomerulonephritis in systemic lupus erythematosus revisited. Kidney International, 65, 521530. doi:10.1111/j.1523-1755.2004.00443.x

[14] Chan, R., Lai, F., Li, E., et al. (2007) Intrarenal cytokine gene expression in lupus nephritis. Annals of the Rheumatic Diseases, 66, 886-892.

[15] Wang, G., Lai, F., Lai, K., et al. (2009) Discrepancy between intrarenal messenger RNA and protein expression of ACE and ACE2 in human diabetic nephropathy. American Journal of Nephrology, 29, 524531. doi:10.1159/000185629

[16] Chan, R., Lai, F., Li, E., et al. (2004) Expression of chemokine and fibrosing factor messenger RNA in the urinary sediment of patients with lupus nephritis. Arthritis Rheum, 50, 2882-2890.

[17] Chan, T., Li, F., Tang, C., et al. (2000) Efficacy of mycophenolate mofetil in patients with diffuse proliferative lupus nephritis. Hong Kong-Guangzhou nephrology study group. The New England Journal of Medicine, 343, 1156-1162. doi:10.1056/NEJM200010193431604

[18] Brunner, H., Mueller, M., Rutherford, C., et al. (2006) Urinary neutrophil gelatinase-associated lipocalin as a biomarker of nephritis in childhood-onset systemic lupus erythematosus. Arthritis Rheum, 54, 2577-2584. doi:10.1002/art.22008

[19] Bolignano, D., Coppolino, G., Campo, S., et al. (2008) Urinary neutrophil gelatinase-associated lipocalin (NGAL) is associated with severity of renal disease in proteinuric patients. Nephrol Dial Transplant, 23, 414-416.

[20] Hinze, C., Suzuki, M., Klein-Gitelman, M., et al. (2009) Neutrophil gelatinase-associated lipocalin is a predictor of the course of global and renal childhood-onset systemic lupus erythematosus disease activity. Arthritis Rheum, 60, 2772-2781. doi:10.1002/art.24751

[21] Machida, H., Ito, S., Hirose, T., et al. (2010) Expression of Toll-like receptor 9 in renal podocytes in childhood-onset active and inactive lupus nephritis. Nephrol Dial Transplant, 25, 2430-2537.

[22] Benigni, A., Caroli, C., Longaretti, L., et al. (2007) Involvement of renal tubular Toll-like receptor 9 in the development of tubulointerstitial injury in systemic lupus. Arthritis Rheum, 56, 1569-1578.

[23] Papadimitraki, E., Tzardi, M., Bertsias, G., et al. (2009) Glomerular expression of toll-like receptor-9 in lupus nephritis but not in normal kidneys: implications for the amplification of the inflammatory response. Lupus, 18, 831-835. doi:10.1177/0961203309103054

[24] Leadbetter, E., Rifkin, I., Hohlbaum, A., et al. (2002) Chromatin-IgG complexes activate $B$ cells by dual engagement of $\operatorname{IgM}$ and Toll-like receptors. Nature, 416, 603-607. doi:10.1038/416603a

[25] Mortensen, E., Fenton, K. and Rekvig, O. (2008) Lupus nephritis: the central role of nucleosomes revealed. American Journal of Pathology, 172, 275-283.

[26] Patole, P., Pawar, R., Lech, M., et al. (2006) Expression and regulation of Toll-like receptors in lupus-like immune complex glomerulonephritis of MRL-Fas(lpr) mice. Nephrol Dial Transplant, 21, 3062-3073. doi:10.1093/ndt/gfl336

[27] Means, T., Latz, E., Hayashi, F., et al. (2005) Human lupus autoantibody-DNA complexes activate DCs through cooperation of CD32 and TLR9. Journal of Clinical Investigation, 115, 407-417. 